\title{
Italian consensus on treatment of spasticity in multiple sclerosis
}

\author{
G. Comi ${ }^{a}$ (iD, A. Solarib (D), L. Leocani ${ }^{a}$, D. Centonze ${ }^{c, d}$ (DD, S. Otero-Romero ${ }^{e}$ (iD) on behalf of the Italian \\ Consensus Group on treatment of spasticity in multiple sclerosis
}

${ }^{\mathrm{a}}$ Institute of Experimental Neurology, Università Vita-Salute, Ospedale S. Raffaele, Milan; ${ }^{\mathrm{b}}$ Unit of Neuroepidemiology, Fondazione IRCCS Istituto Neurologico C. Besta, Milan; ${ }^{\mathrm{c}}$ Unit of Neurology, IRCCS Neuromed, Pozzilli; ${ }^{\mathrm{d}}$ Laboratory of Synaptic

Immunopathology, Department of Systems Medicine, Tor Vergata University, Rome, Italy; and ${ }^{\mathrm{e}} \mathrm{MS}$ Centre of Catalonia (Cemcat), Dept. Neurology/Neuroimmunology, Preventive Medicine Dept., Vall d'Hebron University Hospital, Barcelona, Spain

\section{Keywords:}

botulinum toxin, multiple sclerosis, pharmacological treatment, repetitive transcranial magnetic stimulation, spasticity

Received 4 March 2019 Accepted 27 September 2019

European Journal of Neurology 2020, 27: 445-453

doi:10.1111/ene. 14110

[Correction added on 4 February 2020, after first online publication: The affliations for D. Centonze have been updated.]
Background: Spasticity is a frequent multifactorial manifestation of multiple sclerosis (MS), affecting mostly the chronic courses of the disease. Its impact on patient functioning and quality of life is profound. Treatment of spasticity includes oral and intrathecal anti-spastic drugs, muscle injections with relaxant agents, physical therapy, electrical and magnetic stimulation and peripheral nerve stimulation, alone or in various combinations.

Methods: This Italian consensus on the treatment of spasticity in MS was produced by a large group of Italian MS experts in collaboration with neurophysiologists, experts in the production of guidelines and patients' representatives operating under the umbrella of the Italian Neurological Society, the Associazione Italiana Sclerosi Multipla and the European Charcot Foundation. This guideline was developed in accordance with the Grading of Recommendations Assessment, Development and Evaluation (GRADE) approach. A total of 11 questions were formulated following the PICO framework (patients, intervention, comparator, outcome). Controlled studies only were included in the analysis.

Results: Despite some consistent limitations due to the poor methodological quality of most studies, there was a consensus on a strong recommendation for the use of intrathecal baclofen, oromucosal spray of nabiximols and intramuscular injection of botulinum toxin. The level of recommendation was weak for oral baclofen, tizanidine, gabapentin, benzodiazepines and transcranial magnetic stimulation.

Conclusions: There is a clear need for new larger multicentre well-designed clinical trials with a duration that allows the persistence of the effects and the long-term safety of the interventions to be evaluated.

\section{Introduction}

Spasticity is a very frequent and disabling manifestation of multiple sclerosis (MS), affecting about 60\% of patients [1] and almost all patients in the progressive course of the disease. The clinical manifestations of spasticity can show some variability; the most common findings are muscle stiffness, co-contraction,

Correspondence: G. Comi, Insitute of Experimental Neurology, IRCCS Ospedale San Raffaele, Via Olgettina 48, 20132 Milano, Italy (tel.: + 390226432990; fax: + 390226433746; e-mail: comi.giancarlo@hsr.it).

All authors of the Italian Consensus Group on the treatment of spasticity in multiple sclerosis are listed in Appendix S1. spasms and pain. Spasticity may undergo sudden exacerbations due to infections, noxious stimuli and emotions [2]. The impact on patient functioning and quality of life is profound [3] mobility can be dramatically limited, as well as sleep quality.

Spasticity is assessed using appropriate semi-quantitative scales, such as the Ashworth Scale (AS), the modified Ashworth Scale (MAS) and the Tardieu Scale based on the degree of resistance to passive movement of different body segments as perceived by the examiner [4], or by neurophysiological investigations such as the H-reflex and the Wartenberg pendulum tests [5], mostly used in research. Patient-reported outcomes, such as the Numerical Rating Scale (NRS), the Visual 
Analogue Scale (VAS) and the Multiple Sclerosis Spasticity Scale (MSSS-88) are largely used both in clinical trials and in clinical practice [6] because of the poor responsiveness of the AS and MAS and the complex manifestations of spasticity. They have some limitations because it is quite hard for the patient to recognize the contribution of spasticity amongst the different variables influencing motor abilities.

Reviews and consensus papers have already been produced on the treatment of spasticity in MS [7-11]; however, an up-to-date study that includes pharmacological and non-pharmacological interventions is still lacking. This Italian consensus on the treatment of spasticity in MS was produced by a large group of Italian MS experts in collaboration with neurophysiologists, experts in the production of guidelines, and patients' representatives operating under the umbrella of the Italian Neurological Society, the Associazione Italiana Sclerosi Multipla and the European Charcot Foundation. The aim was to produce a rigorous full systematic review based on the Grading of Recommendations Assessment, Development and Evaluation (GRADE) analysis in order to formulate final recommendations based on the quality of evidence and the balance between advantages and disadvantages of the interventions. Physical therapy was not evaluated in this consensus.

\section{Methods (search strategy and consensus)}

This guideline was developed in accordance with the GRADE working group [12]. A large multidisciplinary working group chaired by GC and integrated by neurologists, methodologists and patients' representatives was established. For operational purposes, the group was divided into three working groups: (i) assessment, coordinated by LL, (ii) interventional, coordinated by DC, and (iii) methodological, coordinated by AS. During preliminary meetings the guideline working group defined the scope of the document, establishing the aspects to be covered and the specific methodology to be used to reach consensus, as well as the general work plan and the distribution of tasks.

A total of 11 questions were formulated following the PICO framework (patients, intervention, comparator, outcome) (Table 1) and an explicit list of outcomes for each question was proposed by the assessment group. Given the complexity and heterogeneity of the outcomes used to assess the efficacy and effectiveness of the spasticity interventions, a specific literature search on the topic was conducted. The assessment group analysed the outcomes used in the literature and produced a list of outcomes that were graded within this working group, according to their relevance from a clinical perspective, using a nine-
Table 1 PICO questions

1. In MS patients with spasticity, is oral baclofen, in comparison with placebo, able to relieve spasticity symptoms and/or signs?

2. In MS patients with spasticity, is intrathecal baclofen superior to oral baclofen in relieving spasticity symptoms and/or signs?

3. In MS patients with spasticity, are cannabinoids more effective than placebo or other interventions in relieving spasticity symptoms and/or signs?

4. In MS patients with spasticity, is botulinum toxin superior to placebo or other interventions in relieving spasticity symptoms and/or signs?

5. In MS patients with spasticity, is tizanidine more effective than placebo in relieving spasticity symptoms and/or signs?

6. In MS patients with spasticity, is gabapentin or pregabalin more effective than placebo or other interventions in relieving spasticity symptoms and/or signs?

7. In MS patients with spasticity, is diazepam or clonazepam more effective than placebo or other interventions in relieving spasticity symptoms and/or signs?

8. In MS patients with spasticity, are aminopyridines more effective than placebo or other interventions in relieving spasticity symptoms and/or signs?

9. In MS patients with spasticity, are peripheral stimulation approaches [such as transcutaneous electrical nerve stimulation (TENS)] more effective than sham stimulation in relieving spasticity symptoms and/or signs?

10. In MS patients with spasticity, is magnetic stimulation more effective than sham stimulation in relieving spasticity symptoms and/or signs?

11. In MS patients with spasticity, is transcranial direct current stimulation (TDCS) more effective than sham stimulation in relieving spasticity symptoms and/or signs?

Table 2 List of outcomes selected for the GRADE analysis

Clinical outcomes
Ashworth Scale
Modified Ashworth Scale
Tardieu Scale
Patient-reported outcomes
Spasticity Numerical Rating Scale (NRS)
MS Spasticity Scale (MSSS-88)
Spasticity Visual Analog Scale (VAS)

point Likert scale. Only outcomes graded as critical or important were included in the PICO questions and considered in the evidence analysis [13] (Table 2).

A systematic literature search was conducted for each of the PICO questions in the Medical Literature Analysis and Retrieval System Online (MEDLINE), following a predefined review protocol and in line with the preferred reporting items for systematic reviews and meta-analysis (PRISMA) statement [14]. The search included studies performed from 1 January 2007 to 16 August 2017. The relevant literature published prior to 2007 was obtained from two high quality systematic reviews [10,11]. All results were reviewed by two independent reviewers according to the inclusion/exclusion criteria specified in Table 3. Since the search was limited to MEDLINE, the only 
Table 3 Inclusion criteria for the literature analysis

Date range: 1 January 2007-16 August 2017

Language: English

Publication type: Clinical study; full text

Study design: Comparative study (e.g. randomized, controlled)

Patient population: at least $50 \%$ of patients with MS

Outcomes: Spasticity outcome(s)

duplicates were between searches, with some studies relevant to multiple searches. Records meeting the inclusion criteria were retrieved for full text review.

The scientific evidence for spasticity interventions was evaluated using the GRADE approach to assess the quality of evidence for each outcome across studies taking into account the following items: study design, risk of bias, inconsistency, indirectness and imprecision [13]. This transparent and structured process was translated into the Evidence Profile and Summary of Findings tables in which the evidence for each outcome across all studies for each PICO question is categorized into 'very low', 'low', 'high' and 'very high' quality and serves as the basis to formulate the clinical recommendations.

The means by which the consensus was produced was based on the opinion of experts and patients. All participants had to read the papers and were exposed to the results of the GRADE analysis. For each of the questions participants were asked to judge if there was evidence for the efficacy of the specific interventions. There were two possibilities only: yes or no. An agreement required at least $80 \%$ of votes in the same direction (yes or no). For the recommendations, there were three different levels: no, weak and strong. Again to reach a given level of recommendation the consensus required the agreement of at least $80 \%$ of the participants. With this methodology the final consensus is based not only on the strength of the evidence of the clinical trials but also on the way the participants interpret the results. The three panels (assessment, interventional and methodological) were involved in the final consensus process for the production of the recommendations. This process was overviewed by the chairman GC with the collaboration of SOR.

\section{Results}

Detailed results of the GRADE analysis for each intervention are provided in Appendix S2.

\section{Oral and intrathecal baclofen}

Question 1: In MS patients with spasticity, is oral baclofen, in comparison with placebo, able to relieve spasticity symptoms and/or signs?
Question 2: In MS patients with spasticity, is intrathecal baclofen superior to oral baclofen in relieving spasticity symptoms and/or signs?

One search was conducted in PubMed regarding oral (question 1) and intrathecal (question 2) baclofen. In addition, articles from the review by Otero-Romero et al. [11] were assessed for relevance. Thirty records were identified through PubMed search. Of these, 28 were excluded after screening for the following reasons: commentary/opinion $(n=2)$, lack of a comparator $(n=2)$, absence of clinical data $(n=2)$, less than $50 \%$ of MS patients included $(n=3)$, different drug/ intervention $(n=5)$, unclear study type $(n=11)$, outcome not related to spasticity $(n=3)$.

A further 16 full text articles were identified through the review article of Otero-Romero et al. [11]. Of these, 10 were excluded for the following reasons: commentary/opinion $(n=1)$, not a specified study type $(n=9)$. Only two articles found in PubMed and six in the review article [11] were therefore assessed for eligibility. Of these articles, two were not included in the GRADE analysis as they did not assess one of the five prioritized spasticity-related outcomes (AS, MAS, spasticity NRS, MSSS-88 and spasticity VAS).

Overall, there were four full text articles included in the GRADE analysis for oral baclofen, from which two outcomes were assessed (AS and spasticity NRS), and two articles for intrathecal baclofen (both assessing AS).

(a) Oral baclofen

Four double-blind, placebo-controlled studies were included in the GRADE analysis [15-18]. Of these, three were crossover studies $[15,16,18]$ and one had an unclear study design [17]. All four studies assessed the Ashworth spasticity scale, whilst the spasticity NRS was also measured in one study [18].

\section{Quality of the evidence and recommendation}

Overall, the quality of the evidence for the studies assessing the AS [15-18] and the spasticity NRS [18] was judged very low; there was a consensus on a weak recommendation for the use of oral baclofen for treatment of spasticity in MS.

(b) Intrathecal baclofen

Two selected double-blind randomized controlled trials assessed the effect of intrathecal baclofen on the Ashworth spasticity scale [19,20].

\section{Quality of the evidence and recommendation}

Although the quality of the evidence for these studies was very low, there was a consensus of the panel to recommend intrathecal baclofen for the treatment of spasticity; the strength of recommendation was strong. 
Six studies were included in the GRADE analysis. The primary end-point was the AS in five studies; all were double-blind, placebo-controlled [5,38-41]; the MAS was the primary end-point in a double-blind, double-dummy, two-way crossover, placebo-controlled randomized controlled trial [42].

\section{Quality of the evidence and recommendation}

There are various limitations in the six examined studies: small sample size [5,41], no details of statistical methods, major statistical problems $[5,38,39,41]$, imprecision (time interval between dosing and assessment varied, and non-significant baseline differences in score were addressed as covariate in analysis) [40], publication bias (support or author affiliation with drug company) [5,38-40,42]. The quality of evidence was classified as low for the MAS and moderate for the AS. There was a consensus for a weak recommendation for the use of tizanidine.

\section{GABAergic drugs (gabapentin)}

Question 6: In MS patients with spasticity, is gabapentin or pregabalin more effective than placebo or other interventions in relieving spasticity symptoms and/or signs?

Two studies, both randomized double-blind, placebo-controlled crossover trials [43,44], met the criteria for inclusion in the systematic review and GRADE analysis. The AS was the pre-specified outcome measure in both randomized controlled trials. Compared to placebo, gabapentin reduced spasticity in both categorical $(P=0.04)$ and continuous analyses (median score reduction of $2.7 ; P=0.007$ ).

\section{Quality of the evidence and recommendation}

Very serious limitations are the very small sample size of the studies and the suboptimal description of the results. The unclear description of outcome in one study ('modified' Ashworth scale) produced serious imprecision [43]. The resulting quality of the evidence was very low. Based on the magnitude of the effect and the good tolerability of the drug (although the treatment period was very short) the panel agreed on a weak recommendation for using gabapentin to reduce spasticity in MS patients.

\section{Benzodiazepines}

Question 7: In MS patients with spasticity, is diazepam or clonazepam more effective than placebo or other interventions in relieving spasticity symptoms and/or signs? 
Three studies met the criteria for inclusion in the systematic review and GRADE analysis [[41,45,46]. Two were crossover randomized controlled trials comparing diazepam to baclofen $[45,46]$, one of which was double-blind [46]. The other study was a randomized controlled trial comparing diazepam to tizanidine [41]. The AS was the pre-specified outcome measure used in the three studies. Diazepam significantly reduced muscle tone; however, there were no significant differences compared to the efficacy of tizanidine and oral baclofen.

\section{Quality of the evidence and recommendation}

Serious limitations are the small sample size of the studies, one of which was not randomized [49], insufficient washout period $[45,46]$, and lack of clarity on the patients analysed [45] and on the statistical methods [41]. Serious imprecision originated from the fact that dosing was not by weight, AS scores were not reported and the table showing improvement scores seemed to mislabel interventions [41]. The resulting quality of the evidence was low.

Based on the effect of oral diazepam which was not superior to the comparator (oral baclofen or tizanidine) and the limited tolerability of the drug, the guideline panel agreed on a weak recommendation for using benzodiazepines to reduce spasticity in MS patients.

\section{Aminopyridines}

Question 8: In MS patients with spasticity, are aminopyridines more effective than placebo or other interventions in relieving spasticity symptoms and/or signs?

Of three records identified, two were excluded because they were review papers; one paper was assessed in full [47]. The paper reported the results of a double-blind, placebo-controlled parallel group trial testing the efficacy and safety of fampridine on muscle strength. The paper was excluded because spasticity was not the target of the intervention and none of the accepted spasticity outocmees were used.

No GRADE analysis was performed.

\section{Transcutaneous electrical nerve stimulation (TENS)}

Question 9: In MS patients with spasticity, are peripheral stimulation approaches (such as TENS) more effective than sham stimulation in relieving spasticity symptoms and/or signs?

Fifteen records were identified through PubMed search; 10 were excluded and five full text articles were assessed for eligibility. Four did not meet the inclusion criteria for various methodological reasons or because the articles were systematic reviews, so one article only was included in the GRADE analysis [48]. The study compared the relative efficacy of baclofen and self-applied TENS for the treatment of spasticity in the lower extremities in MS. The primary outcome was the MAS. Spasticity decreased significantly in both groups, with a larger effect in the TENS group.

\section{Quality of the evidence and recommendation}

There were very serious limitations: unblinded study, small sample size, short treatment and follow-up, high loss to follow-up, no discussion of confounders, and no control of the administration of TENS because the treatment was performed at home. There was some risk of inconsistency because of a possible mismatch between author conclusion and data provided regarding which treatment had a superior effect. The quality of the evidence was considered to be very low. There was a consensus in the panel that from the low quality of the evidence TENS cannot be recommended as a treatment of spasticity in MS.

\section{Repetitive transcranial magnetic stimulation (rTMS)}

Question 10: In MS patients with spasticity, is magnetic stimulation more effective than sham stimulation in relieving spasticity symptoms and/or signs?

Repetitive transcranial magnetic stimulation (rTMS) is a non-invasive technique that induces changes in cortical excitability at the site of stimulation and at distant sites, leading to either facilitation or inhibition depending on the frequency and pattern of pulses. The effects of rTMS are possibly mediated through the induction of short-term and long-term synaptic potentiation mechanisms, opening the possibility of therapeutic applications [49]. Repetitive transcranial theta burst stimulation is a recently developed high frequency and short duration rTMS protocol, able to induce more long-lasting effects [50].

PubMed search identified 12 references regarding repetitive magnetic stimulation. In total, five studies [51-55] fulfilled the inclusion criteria for this review. Four major outcomes were used in the studies: the MAS [51-54], the AS [55], the MSSS-88 [54] and the spasticity VAS [51]. Three study designs were double-blind sham-controlled, randomized controlled trials [51,54,55] and two were pseudo-randomized sham-controlled studies [52,53]. Concerning the MAS, a treatment effect was observed with real but not sham stimulation in three of four studies [5254], whilst one study [51] found improvement of the 
MAS in both groups, with no significant difference between groups. In the fifth study [55] a combined score including the AS showed a significant benefit of real versus sham stimulation; the positive result of the primary end-point was supported by a positive effect on a numerical rating scale of daily activities.

\section{Quality of the evidence and recommendation}

For all the studies the quality of the evidence can be classified as very low because of many limitations. All studies had very small sample sizes; two studies were quasi-randomized limiting the initial quality of the evidence [52,53]; a treatment effect not consistently shown across spasticity measures [51,54]; there was a significant difference in baseline clinical scores of spasticity between groups [55]; the AS was part of a composite clinical score [58]. Inconsistencies emerged in some studies [51,54].

The panel agreed to a weak recommendation of brain transcranial magnetic stimulation for the treatment of spasticity in MS.

\section{Transcranial direct current stimulation (TDCS)}

Question 11: In MS patients with spasticity, is TDCS more effective than sham stimulation in relieving spasticity symptoms and/or signs?

Transcranial direct current stimulation (TDCS) is another simple, non-invasive technique that can induce sustained excitability changes in relatively restricted human brain areas [56], modulating spontaneous neuronal firing rates, synaptic and non-synaptic plasticity [57].

For the effects of TDCS on MS spasticity, 13 papers were identified. Twelve were excluded for various reasons: outcomes not related to spasticity $(n=2)$, different drug/intervention $(n=6)$, not a specified study type $(n=3)$, review $(n=1)$. One study only was included in the GRADE analysis [58].

The included study was a single-centre, randomized, double-blind, sham-controlled trial with the MAS as primary end-point. No significant effects of TDCS emerged.

\section{Quality of the evidence and recommendation}

The study has major limitations, including the small sample size, imprecision (only one side studied in each patient) and technical issues as TDCS was specifically applied over the motor cortex corresponding to the more affected leg. The panel concluded for the absence of evidence to recommend TDCS to treat spasticity in MS patients.

\section{Conclusions}

Spasticity is a frequent symptom of MS, particularly in the progressive phase of the disease, resulting in a strong impact on quality of life and working capacity. It is a complex phenomenon with interaction with other MS symptoms and signs such as pain, weakness and ataxia. As a consequence, the assessment of spasticity is quite difficult, particularly in the area of patient-reported outcome, explaining the wide variety of approaches taken to assess this symptom. On the other hand, the measures of spasticity, such as the AS and MAS, the most commonly used in clinical trials, require some collaboration of the patient; moreover, they are characterized by a low responsiveness, in addition to their low sensitivity. The combination of these limiting factors explains the large inter- and intra-individual variability in the responses to therapeutic interventions. Overall, the methodological quality of the studies exploring interventions on spasticity affecting MS patients was poor. The sample sizes were small in all studies, with the exception of some studies evaluating the effects of cannabinoids, and the duration of the trials never exceeded 3 weeks, which limits inference of long-term efficacy. Other factors with negative impact on the quality of the studies were marked heterogeneity in patients' characteristics, the monocentric design and the variability of treatment regimens. Despite all these consistent limitations, which should be carefully considered when evaluating the results of clinical trials, some interventions demonstrated a significant reduction of spasticity, with a good internal consistency in some studies. The level of recommendation was weak for most of the examined interventions, with the exception of a strong recommendation for intrathecal baclofen, cannabinoids and botulinum toxin. In our consensus interventions in the area of physical therapy were not included. Despite a lack of evidence, stretching is commonly used and its role is supported by a recent study [59]. At present spasticity is frequently undertreated and monitoring of interventions is quite poor. As far as is known no treatment algorithms have been validated. There is a clear need for new larger multicentre well-designed clinical trials with a duration that allows the persistence of the effects and long-term safety of the interventions to be evaluated. The sequencing and the combination of interventions should also be investigated. On the other hand, our assessment and monitoring of spasticity should be improved, expanding the role of the patient perspective, to better utilize the already available interventions and to test new potential therapies. 


\section{Acknowledgements}

This paper was supported by the European Charcot Foundation.

Italian Consensus Group on the treatment of spasticity in multiple sclerosis: Roberta Amadeo, Maria Pia Amato, Antonio Bertolotto, Laura Boffa, Giampaolo Brichetto, Diego Centonze, Giancarlo Comi, Mauro Comola, Angelo Ghezzi, Letizia Leocani, Giacomo Lus, Maria Giovanna Marrosu, Franco Molteni, Susana Otero-Romero, Francesco Patti, Carlo Pozzilli, Marco Rovaris, Francesco Saccà, Edoardo Sessa, Alessandra Solari, Claudio Solaro, Maria Trojano, Carlo Trompetto, Mauro Zaffaroni.

\section{Disclosure of conflicts of interest}

The authors declare no financial or other conflicts of interest.

\section{Ethical approval}

Study approval of any board or ethics committee was not required since this is a consensus project reviewing and analysing data from other studies and by literature search and not a clinical trial or research project involving patients or healthy subjects.

\section{Consent}

Informed consent was not required since this is a consensus project reviewing and analysing data from other studies and by literature search and not a clinical trial or research project involving patients or healthy subjects.

\section{Supporting Information}

Additional Supporting Information may be found in the online version of this article:

Appendix S1. Italian consensus group on the treatment of spasticity in multiple sclerosis.

Appendix S2. Detailed results of the paper analysis.

\section{References}

1. Smith CR, LaRocca NG, Giesser BS, Scheinberg LC. High-dose oral baclofen: experience in patients with multiple sclerosis. Neurology 1991; 41: 1829-1831.

2. Haselkorn JK, Balsdon Richer C, Fry Welch D, et al. Overview of spasticity management in multiple sclerosis. Evidence-based management strategies for spasticity treatment in multiple sclerosis. J Spinal Cord Med 2005; 28: $167-199$.
3. Milinis K, Tennant A, Young CA, TONiC Study Group. Spasticity in multiple sclerosis: associations with impairments and overall quality of life. Mult Scler Relat Disord 2016; 5: 34-39.

4. Platz T, Eickhof C, Nuyens G, Vuadens P. Clinical scales for the assessment of spasticity, associated phenomena, and function: a systematic review of the literature. Disabil Rehabil 2005; 27: 7-18.

5. Emre M, Leslie GC, Muir C, et al. Correlations between dose, plasma concentrations, and antispastic action of tizanidine (Sirdalud). J Neurol Neurosurg Psychiatry 1994; 57: 1355-1359.

6. Hobart JC, Riazi A, Thompson AJ, et al. Getting the measure of spasticity in multiple sclerosis: the Multiple Sclerosis Spasticity Scale (MSSS-88). Brain 2006; 129: 224-234.

7. Rizzo MA, Hadjimichael OC, Preiningerova J, Vollmer TL. Prevalence and treatment of spasticity reported by multiple sclerosis patients. Mult Scler 2004; 10: 589-595.

8. Thompson AJ, Jarrett L, Lockley L, et al. Clinical management of spasticity. J Neurol Neurosurg Psychiatry 2005; 76: 459-463.

9. Lapeyre E, Kuks JBM, Meijler WJ. Spasticity: revisiting the role and the individual value of several pharmacological treatments. NeuroRehabilitation 2010; 27: 193200 .

10. Amatya B, Khan F, La Mantia L, et al. Non pharmacological interventions for spasticity in multiple sclerosis. Cochrane Database Syst Rev 2013; (2): CD009974.

11. Otero-Romero S, Sastre-Garriga J, Comi G, et al. Pharmacological management of spasticity in multiple sclerosis: systematic review and consensus paper. Mult Scler 2016; 22: 1386-1396.

12. Guyatt G, Oxman AD, Akl EA, et al. GRADE guidelines: 1. Introduction - GRADE evidence profiles and summary of findings tables. J Clin Epidemiol 2011; 64: 383-394.

13. Leone MA, Brainin M, Boon $\mathrm{P}$, et al. Guidance for the preparation of neurological management guidelines by EFNS scientific task forces - revised recommendations 2012. Eur J Neurol 2013; 20: 410-419.

14. Moher D, Liberati A, Tetzlaff J, et al. Preferred reporting items for systematic reviews and meta-analyses: the PRISMA statement. PLoS Med 2009; 6: e1000097.

15. Brar SP, Smith MB, Nelson LM, et al. Evaluation of treatment protocols on minimal to moderate spasticity in multiple sclerosis. Arch Phys Med Rehabil 1991; 72: 186-189.

16. Hudgson P, Weightman D. Baclofen in the treatment of spasticity. Br Med J 1971; 4: 15-17.

17. Levine IM, Jossmann PB, DeAngelis V. Liorseal, a new muscle relaxant in the treatment of spasticity - a double-blind quantitative evaluation. Dis Nerv Syst 1977; 38: 1011-1015.

18. Orsnes GB, Sørensen PS, Larsen TK, Ravnborg M. Effect of baclofen on gait in spastic MS patients. Acta Neurol Scand 2000; 101: 244-248.

19. Penn RD, Savoy SM, Corcos D, et al. Intrathecal baclofen for severe spinal spasticity. $N$ Engl J Med 1989; 320: 1517-1521.

20. Middel B, Kuipers-Upmeijer H, Bouma J, et al. Effect of intrathecal baclofen delivered by an implanted programmable pump on health related quality of life in 
patients with severe spasticity. J Neurol Neurosurg Psychiatry 1997; 63: 204-209.

21. Collin C, Davies P, Mutiboko IK, et al. Randomized controlled trial of cannabis-based medicine in spasticity caused by multiple sclerosis. Eur J Neurol 2007; 14: 290-296.

22. Killestein J, Hoogervorst ELJ, Reif M, et al. Safety, tolerability, and efficacy of orally administered cannabinoids in MS. Neurology 2002; 58: 1404-1407.

23. van Amerongen G, Kanhai K, Baakman AC, et al. Effects on spasticity and neuropathic pain of an oral formulation of $\Delta 9$-tetrahydrocannabinol in patients with progressive multiple sclerosis. Clin Ther 2018; 40: 1467-1482.

24. Vaney C, Heinzel-Gutenbrunner M, Jobin P, et al. Efficacy, safety and tolerability of an orally administered cannabis extract in the treatment of spasticity in patients with multiple sclerosis: a randomized, double-blind, placebo-controlled, crossover study. Mult Scler 2004; 10: 417-424.

25. Zajicek J, Fox P, Sanders H, et al. Cannabinoids for treatment of spasticity and other symptoms related to multiple sclerosis (CAMS study): multicentre randomised placebo-controlled trial. Lancet 2003; 362: $1517-1526$.

26. Zajicek JP, Sanders HP, Wright DE, et al. Cannabinoids in multiple sclerosis (CAMS) study: safety and efficacy data for 12 months follow up. J Neurol Neurosurg Psychiatry 2005; 76: 1664-1669.

27. Collin C, Ehler E, Waberzinek G, et al. A double-blind, randomized, placebo-controlled, parallel-group study of Sativex, in subjects with symptoms of spasticity due to multiple sclerosis. Neurol Res 2010; 32: 451-459.

28. Leocani L, Nuara A, Houdayer E, et al. Sativex(®) and clinical-neurophysiological measures of spasticity in progressive multiple sclerosis. J Neurol 2015; 262: 2520 2527.

29. Notcutt W, Langford R, Davies P, et al. A placebo-controlled, parallel-group, randomized withdrawal study of subjects with symptoms of spasticity due to multiple sclerosis who are receiving long-term Sativex ${ }^{\circledR}$ (nabiximols). Mult Scler 2012; 18: 219-228.

30. Novotna A, Mares J, Ratcliffe S, et al. A randomized, double-blind, placebo-controlled, parallel-group, enriched-design study of nabiximols* (Sativex( $(\circledR)$ ), as add-on therapy, in subjects with refractory spasticity caused by multiple sclerosis. Eur J Neurol 2011; 18: 1122-1131.

31. Wade DT, Makela P, Robson P, et al. Do cannabisbased medicinal extracts have general or specific effects on symptoms in multiple sclerosis? A double-blind, randomized, placebo-controlled study on 160 patients. Mult Scler 2004; 10: 434-441.s

32. Ball S, Vickery J, Hobart J, et al. The Cannabinoid Use in Progressive Inflammatory brain Disease (CUPID) trial: a randomised double-blind placebo-controlled parallel-group multicentre trial and economic evaluation of cannabinoids to slow progression in multiple sclerosis. Health Technol Assess 2015; 19(12): 1-187. vii-viii, xxvxxxi.

33. Zajicek JP, Hobart JC, Slade A, et al. Multiple sclerosis and extract of cannabis: results of the MUSEC trial. $J$ Neurol Neurosurg Psychiatry 2012; 83: 1125-1132.

34. Hyman N, Barnes M, Bhakta B, et al. Botulinum toxin (Dysport) treatment of hip adductor spasticity in multiple sclerosis: a prospective, randomised, double blind, placebo controlled, dose ranging study. J Neurol Neurosurg Psychiatry 2000; 68: 707-712.

35. Snow BJ, Tsui JK, Bhatt MH, et al. Treatment of spasticity with botulinum toxin: a double-blind study. Ann Neurol 1990; 28: 512-515.

36. Giovannelli M, Borriello G, Castri P, et al. Early physiotherapy after injection of botulinum toxin increases the beneficial effects on spasticity in patients with multiple sclerosis. Clin Rehabil 2007; 21: 331-337.

37. Paoloni M, Giovannelli M, Mangone M, et al. Does giving segmental muscle vibration alter the response to botulinum toxin injections in the treatment of spasticity in people with multiple sclerosis? A single-blind randomized controlled trial. Clin Rehabil 2013; 27: 803-812.

38. A double-blind, placebo-controlled trial of tizanidine in the treatment of spasticity caused by multiple sclerosis. United Kingdom Tizanidine Trial Group. Neurology 1994; 44: S70-78.

39. Nance PW, Sheremata WA, Lynch SG, et al. Relationship of the antispasticity effect of tizanidine to plasma concentration in patients with multiple sclerosis. Arch Neurol 1997; 54: 731-736.

40. Smith C, Birnbaum G, Carter JL, et al. Tizanidine treatment of spasticity caused by multiple sclerosis: results of a double-blind, placebo-controlled trial. US Tizanidine Study Group. Neurology 1994; 44: S34-S42; discussion S42-43.

41. Rinne UK. Tizanidine treatment of spasticity in multiple sclerosis and chronic myelopathy. Curr Ther Res Clin Exp 1980; 28: 827-836.

42. Vakhapova V, Auriel E, Karni A. Nightly sublingual tizanidine $\mathrm{HCl}$ in multiple sclerosis: clinical efficacy and safety. Clin Neuropharmacol 2010; 33: 151-154.

43. Mueller ME, Gruenthal M, Olson WL, Olson WH. Gabapentin for relief of upper motor neuron symptoms in multiple sclerosis. Arch Phys Med Rehabil 1997; 78: 521-524.

44. Cutter NC, Scott DD, Johnson JC, Whiteneck G. Gabapentin effect on spasticity in multiple sclerosis: a placebo-controlled, randomized trial. Arch Phys Med Rehabil 2000; 81: 164-169.

45. Cartlidge NE, Hudgson P, Weightman D. A comparison of baclofen and diazepam in the treatment of spasticity. J Neurol Sci 1974; 23: 17-24.

46. From A, Heltberg A. A double-blind trial with baclofen (Lioresal) and diazepam in spasticity due to multiple sclerosis. Acta Neurol Scand 1975; 51: 158-166.

47. Jensen HB, Nielsen JL, Ravnborg M, et al. Effect of slow release-Fampridine on muscle strength, rate of force development, functional capacity and cognitive function in an enriched population of MS patients. A randomized, double blind, placebo controlled study. Mult Scler Relat Disord 2016; 10: 137-144.

48. Shaygannejad V, Janghorbani M, Vaezi A, et al. Comparison of the effect of baclofen and transcutaneous electrical nerve stimulation for the treatment of spasticity in multiple sclerosis. Neurol Res 2013; 35: 636-641.

49. Wassermann EM, Lisanby SH. Therapeutic application of repetitive transcranial magnetic stimulation: a review. Clin Neurophysiol 2001; 112: 1367-1377.

50. Huang Y-Z, Edwards MJ, Rounis E, et al. Theta burst stimulation of the human motor cortex. Neuron 2005; 45: 201-206. 
51. Boutière C, Rey C, Zaaraoui W, et al. Improvement of spasticity following intermittent theta burst stimulation in multiple sclerosis is associated with modulation of resting-state functional connectivity of the primary motor cortices. Mult Scler 2017; 23: 855-863.

52. Centonze D, Koch G, Versace V, et al. Repetitive transcranial magnetic stimulation of the motor cortex ameliorates spasticity in multiple sclerosis. Neurology 2007; 68: $1045-1050$.

53. Mori $\mathrm{F}$, Codecà $\mathrm{C}$, Kusayanagi $\mathrm{H}$, et al. Effects of intermittent theta burst stimulation on spasticity in patients with multiple sclerosis. Eur J Neurol 2010; 17: 295-300.

54. Mori F, Ljoka C, Magni E, et al. Transcranial magnetic stimulation primes the effects of exercise therapy in multiple sclerosis. J Neurol 2011; 258: 1281-1287.

55. Nielsen JF, Sinkjaer T, Jakobsen J. Treatment of spasticity with repetitive magnetic stimulation; a double- blind placebo-controlled study. Mult Scler 1996; 2: 227 232.

56. Stagg CJ, Nitsche MA. Physiological basis of transcranial direct current stimulation. Neuroscientist 2011; 17: $37-53$.

57. Nitsche MA, Fricke K, Henschke U, et al. Pharmacological modulation of cortical excitability shifts induced by transcranial direct current stimulation in humans. $J$ Physiol 2003; 553: 293-301.

58. Iodice R, Dubbioso R, Ruggiero L, et al. Anodal transcranial direct current stimulation of motor cortex does not ameliorate spasticity in multiple sclerosis. Restor Neurol Neurosci 2015; 33: 487-492.

59. Hugos CL, Bourdette D, Chen Y, et al. A group-delivered self-management program reduces spasticity in people with multiple sclerosis: a randomized, controlled pilot trial. Mult Scler J Exp Transl Clin 2017; 3: 2055217317699993. 